\title{
BMJ Open Does smoking reduction worsen mental health? A comparison of two observational approaches
}

\author{
Gemma Taylor, ${ }^{1,2}$ Amy Taylor, ${ }^{2,3,4}$ Marcus R Munafò, ${ }^{2,3,4}$ Ann McNeill, ${ }^{2,5}$ \\ Paul Aveyard ${ }^{2,6}$
}

To cite: Taylor G, Taylor A, Munafò MR, et al. Does smoking reduction worsen mental health? A comparison of two observational approaches. BMJ Open 2015;5: 0007812. doi:10.1136/bmjopen-2015007812

Received 31 January 2015 Revised 21 April 2015 Accepted 23 April 2015

\section{- Prepublication history and additional material is available. To view please visit the journal (http://dx.doi.org/ 10.1136/bmjopen-2015- 007812).}

\section{ABSTRACT}

Objectives: The association between smoking reduction and mental health is of particular interest given that many smokers report that smoking offers mental health benefits. We aimed to assess the association between smoking reduction and change in mental health using two different analytical approaches to determine if there was any evidence of an association. There were no prior hypotheses.

Design: A secondary analysis of prospective individual level patient data from 5 merged placebo-controlled randomised trials of nicotine replacement therapy for smoking reduction.

Participants: All participants were adult smokers, selected because they wanted to reduce but not stop smoking, and had smoked for at least 3 years.

Participants were excluded if they were pregnant, breastfeeding, under psychiatric care, deemed to be unfit by a general practitioner, or part of a cessation programme. 2066 participants were enrolled in the trials, 177 participants were biologically validated as prolonged reducers, and 509 as continuing smokers at both 6-week and 18-week follow-ups.

Primary outcome: Change in mental health from baseline to an 18-week follow-up was measured using the emotional well-being subscale on the Short Form Health Survey-36.

Results: After adjustment for confounding variables, the differences for reducers compared with continuing smokers were: regression modelling $-0.6(95 \% \mathrm{Cl}$ -4.4 to 3.2$)$ and propensity score matching 1.1 (95\% Cl -2.0 to 4.1$)$.

Conclusions: Smoking reduction, sustained for at least 12 weeks, was not associated with change in mental health, suggesting that reducing smoking was no better or worse for mental health than continuing smoking. Clinicians offering smoking reduction as a route to quit can be confident that, on average, smoking reduction is not associated with negative change in mental health.

For numbered affiliations see end of article.

Correspondence to Dr Gemma Taylor; gmjtaylor@gmail.com

\section{INTRODUCTION}

In the UK and in some other countries, smoking reduction is promoted as a route to quit or as a harm reduction approach for

\section{Strengths and limitations of this study}

- The largest study, to date, examining the association between smoking reduction and change in mental health.

- Use of a psychometrically sound mental health measure, which is sensitive to change in mental health.

- Use of propensity score matching to reduce confounding and bias from group membership.

- Study presents a low risk of bias according to the Newcastle-Ottawa Scale for Quality Assessment of Observational Studies.

- Loss to follow-up was high, although the rate was similar to other studies of smoking interventions.

smokers who may not be ready to stop smoking immediately, or who cannot stop. There is limited evidence that reduction itself improves health, ${ }^{2}$ but clearer evidence that people who are supported to reduce are more likely to achieve cessation. ${ }^{3}$

A significant proportion of smokers in countries with mature tobacco epidemics report mental health problems. ${ }^{4}$ Moreover, smokers with and without mental health problems report that smoking offers mental health benefits. ${ }^{5-10}$ Therefore, the effect of smoking reduction on mental health is a particular concern. There is strong evidence that stopping smoking is associated with improved mental health, and this improvement may result from breaking the tobacco withdrawal cycle. ${ }^{11}$ However, it is not clear whether reducing daily cigarette consumption is associated with change in mental health. Two previous studies have reported no significant association between smoking reduction and mental health; although, both were small and therefore may have been insufficiently powered to detect associations, and only one used a standardised measure of mental health. ${ }^{12} 13$ 
No previous literature has discussed the possible impact of smoking reduction on mental health and how this may differ from abrupt cessation; however, based on what is currently known about tobacco and mental health, there are three possibilities. As the chemical components in tobacco have a short half-life, psychological withdrawal symptoms occur shortly after smoking a cigarette, and these symptoms become more severe with longer periods of abstinence. ${ }^{14} 15$ Thus, the first possibility is that by reducing cigarette consumption and, in turn, increasing the duration between cigarettes, the smoker may experience longer periods of and more intense withdrawal symptoms such as irritability, depressed mood and anxiety, thus leading to worsened mental health. Mental health may also worsen for some smokers on reduction if they derive a mental health benefit from smoking, according to the self-medication hypothesis. ${ }^{16}$ Second, there is evidence that higher levels of tobacco consumption are associated with poorer mental health, ${ }^{17} 18$ and that stopping smoking is associated with improved mental health. ${ }^{11}$ Therefore, it follows that if there is a dose-response association between tobacco consumption and mental health, reducing tobacco consumption may be associated with improvements in mental health. The third hypothesis is that mental health could remain unchanged. After maintaining reduced cigarette consumption, the smoker may adapt to their new tobacco consumption level and their mental health will remain the same. Another reason it may not change is that the association between poor mental health and smoking could be caused by a common factor ${ }^{19}$ which would not be expected to change on smoking reduction.

If smoking reduction worsens mental health, this may not be the most appropriate route to quit for those with mental health disorders or who are experiencing stress. However, if smoking reduction improves mental health or leads to no change, smokers and clinicians can be reassured that the intervention is unlikely to cause psychological distress. No previous study has adequately assessed the association, therefore in this study, we aimed to do so. A randomised study to assess this hypothesis would be ideal, however, it would not be practical. Observational methods are useful in this situation and different statistical approaches offer different benefits and drawbacks, therefore, we aimed to compare two common methods to assess the association in question. We conducted a secondary analysis of individual level patient data of five randomised trials of treatment for smoking reduction. We aimed first to estimate the association using traditional regression modelling with adjustment for covariates, and second, to compare the results derived from the regression model with those derived from propensity score matching (PSM). Effect estimates from PSM provide evidence that an association is not due to membership bias or observed confounding, and therefore offer stronger evidence of a causal association. ${ }^{20}$ We compared results from these approaches to determine if there was evidence of an association between smoking reduction and change in mental health.

\section{METHOD}

This study followed STROBE reporting guidelines for observational studies. ${ }^{22}$ PSM procedures were conducted and reported following criteria outlined by a review of PSM methodology. ${ }^{23}$

\section{Study design}

This was a secondary analysis of prospective individual level patient data from five merged randomised, placebo-controlled trials (RCTs) of nicotine replacement therapy (NRT) for smoking reduction. The trials were carried out to a consistent protocol, in which participants were encouraged to reduce their smoking as much as possible with cessation as the end goal and were not offered any mood management.

\section{Setting}

These data were provided by McNeil pharmaceutical company (see reports of trials for further details $\mathrm{K}$ Haustein. A double-blind, randomized, placebocontrolled multicentre trial of a nicotine chewing gum in smoking reduction. Study ID 980-CHC-9021-0013, unpublished data, 2001 and refs 24-27). The trials were conducted in university and medical centres in Denmark, Switzerland, Australia, the USA and Germany.

\section{Study size}

There were 2066 participants enrolled in the trials, which took place between 1997 and 2003. In total, 1035 participants provided smoking data at both 6-week and 18-week follow-ups (figure 1). Notably, these attrition rates were similar to other trials of NRT. ${ }^{28}$

\section{Participants}

All participants were adult smokers (aged $\geq 18$ years), selected because they wanted to reduce but not stop smoking, and had smoked for at least 3 years.

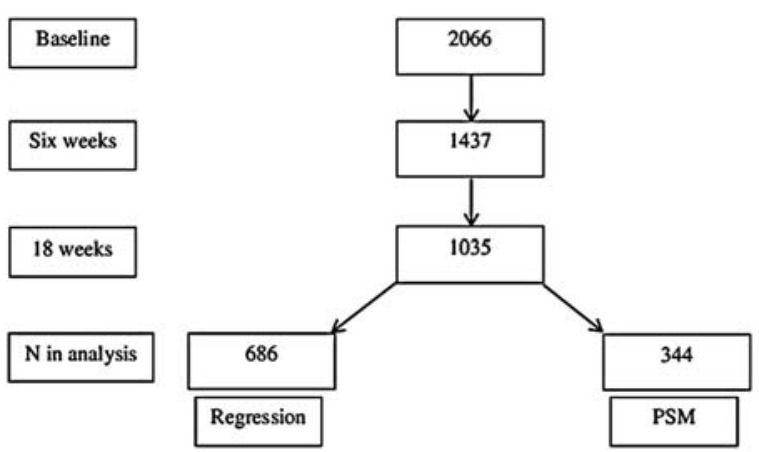

Figure 1 Flow chart of $\mathrm{N}$ participants reporting smoking data and included in each analysis (PSM, propensity score matching). 
Participants were excluded if they were pregnant, breastfeeding, under psychiatric care, deemed to be unfit by a general practitioner, or part of a cessation programme.

At baseline, investigators gathered data on participants' demographic details, age started smoking, cigarettes per day (CPD), nicotine dependence (Fagerström Test of Nicotine Dependence; FTND), ${ }^{29}$ intention to reduce, intention to quit, smoking history (eg, number of previous attempts reduce, longest period without smoking), self-rated effects from smoking ('Relief from smoking questionnaire') (K Haustein, unpublished data, 2001), mental health (measured by the emotional wellbeing subscale on the Short Form Health Survey-36 $\left(\right.$ SF-36/RAND-36) ${ }^{30-32}$ ) (see online supplementary material for further details of variables). To preserve anonymity, some demographic data were unavailable for this secondary analysis.

Participants were followed up to 8-10 times over 12 years, and on each occasion investigators collected data on CPD, recorded an expired air carbon monoxide (CO) reading, and measured quality of life and mental health using the SF-36.

\section{Exposure}

We classified a participant as having achieved prolonged $50 \%$ reduction if they reported at least halving their daily consumption, and a reduction in exhaled CO of at least 1 part per million ( $\mathrm{ppm}$ ) from the baseline measurements at both 6-week and 18-week follow-ups. ${ }^{25-27} 33$ We classified a participant as smoking continuously (ie, a non-reducer) if they reported smoking at both times without achieving a 50\% reduction. Anyone not meeting either definition was excluded from the analysis (ie, participants who quit completely).

\section{Outcome}

The primary outcome was change in mental health from baseline (when all participants were smoking) to 18-week follow-up (after at least 12 weeks of prolonged reduction or continued smoking).

Scores on the SF-36 mental health subscale range from 0 to 100 . In the US general population, the subscale mean and SD are 70.4 (22.0), and scores of $\leq 38$ indicate a probable mental health problem. ${ }^{30-32}$ A minimum clinically important difference (MCID) on this mental health subscale has been defined as a minimum difference between 2.0 and 6.2 points among exposure groups. $^{32}$

\section{Analysis}

All analyses were conducted using Stata V.13. If participants were missing any relevant data they were excluded from the analyses.

Linear regression - In the first analysis, we created a dummy variable representing reduction (reducers=' 1 ', smokers='0'), and used linear regression modelling to examine the association between smoking reduction and mental health at final follow-up with adjustment for baseline mental health values. Change scores were not compared between groups because of regression to the mean when using within-person repeated measures data. Using change scores can overestimate the effect, therefore, it is recommended to use outcome scores with adjustment for baseline values. ${ }^{34}$ We then repeated the model adjusting for FTND score and treatment allocation (active/placebo), age, sex and trial. We also tested for an interaction between treatment allocation and reduction status, to account for the possible effect of active NRT on psychological withdrawal symptoms in reducers. ${ }^{35}$

Propensity score matching-This involved three steps. First, a logistic regression model was developed using covariates which predicted whether or not smokers reduced smoking. Nicotine dependence (FTND) and treatment allocation ${ }^{36}$ were forced into the model, and a forward stepwise procedure was used to add baseline mental health, intention to reduce (eg, make a serious attempt to reduce smoking in 1 month), intention to quit, sex, age, smoking history, CPD, single item questions from the self-rated 'Relief from smoking questionnaire' (eg, report of calming effects from smoking), all at the $\mathrm{p}<0.10$ level. ${ }^{37}$ Second, this model was combined using the PSMATCH2 command in Stata V.13 to calculate each participant's propensity score. Reducers were matched to the continuing smoker in the same trial with the closest propensity score on a ratio of 1:1 using a nearest neighbour greedy algorithm with no replacements, and matching was restricted to the common support region. Third, we conducted a series of model adequacy checks (see online supplementary material). Finally, to determine the association between smoking reduction and mental health we repeated the linear regression model discussed above using the matched sample.

We developed three sensitivity analyses using different PSM models. Our adjusted regression model was rerun for each PSM sensitivity analysis, and we compared the regression coefficients between the sensitivity models. The trials measured key baseline variables consistently; each trial also measured some variables differently to others. Therefore, we (1) matched participants within trials using all relevant variables, (2) matched participants across trials including variables measured consistently and (3) repeated models with and without common support restrictions (see online supplementary material).

\section{RESULTS}

Baseline characteristics of participants in whole sample

Trial non-completers $(n=1342)$ did not differ greatly from those who were included in the analysis (table 1). In total 179 participants were biologically validated as prolonged reducers at both 6 and 18 weeks, and 545 as continuing smokers at both follow-ups. No reducers and six smokers had missing baseline mental health scores, 
Table 1 Baseline characteristics of whole sample

\begin{tabular}{|c|c|c|c|}
\hline & Smokers $(n=509)$ & Reducers $(n=177)$ & Non-completers $(n=1342)^{\star}$ \\
\hline Age, mean (SD) & $45.2(10.6)$ & $46.6(10.9)$ & $43.2(10.9)$ \\
\hline Sex, \% male $(n)$ & $41(229)$ & $42(75)$ & $45(610)$ \\
\hline FTND, M (SD) & $6.0(2.0)$ & $5.9(2.0)$ & $6.2(2.0)$ \\
\hline SF-36 mental health, mean (SD) & $72.6(16.7)$ & $73.7(16.8)$ & $72.3(16.5)$ \\
\hline Treatment status, \% received active $(n)$ & $46(232)$ & $63(112) \dagger$ & 50 (669) \\
\hline
\end{tabular}

and two reducers and 30 smokers had missing mental health data at follow-up. The mean baseline mental health scores of those excluded $(\mathrm{M}=66.7, \mathrm{SD}=19.0)$ and those included $(\mathrm{M}=72.6, \mathrm{SD}=16.8)$ indicated that both groups were psychologically healthy according to the SF-36 cut-off. $^{30-32}$ After exclusion for missing mental health data, 177 participants were biologically validated as reducers and 509 as continuing smokers (figure 1). Table 1 displays baseline characteristics of unmatched smokers and reducers. The proportion of participants who received active treatment was greater among reducers than smokers $(\mathrm{p}<0.0001)$.

\section{Linear regression modelling}

Association between smoking reduction and change in mental health-mental health scores showed a small improvement from baseline to 18-week follow-up in both groups. The mean change $(\mathrm{SD})$ in the reducer group was 0.4 (15.7) compared with 1.0 (15.3) in the continuing smoker group. After adjustment for baseline mental health values, the difference between groups was -0.1 (95\% CI -2.5 to $2.3, \mathrm{p}=0.93)$, indicating that reducers showed a small decline in mental health compared with continuing smokers, however, there was no strong evidence for a difference between groups. After further adjustment for FTND, treatment status, age, sex, treatment status $\times$ reduction status, and trial, the difference between groups was $-0.6(95 \%$ CI -4.4 to $3.2, p=0.75)$. There was no evidence for an interaction between active treatment and reduction status.

Table 2 Baseline characteristics of propensity score-matched sample

\begin{tabular}{lcc}
\hline Variable & $\begin{array}{l}\text { Smokers } \\
(\mathbf{n}=172)\end{array}$ & $\begin{array}{l}\text { Reducers } \\
(\mathbf{n}=172)\end{array}$ \\
\hline Age, mean (SD) & $45.0(10.7)$ & $46.7(10.7)$ \\
Sex, \% male (n) & $47(81)$ & $43(74)$ \\
FTND, M (SD) & $5.81(2.1)$ & $5.9(2.0)$ \\
SF-36 mental health, mean (SD) & $70.8(15.7)$ & $73.9(16.6)$ \\
Treatment status, \% received & $60(104)$ & $62(107)$ \\
active (n) & & \\
\hline FTND, Fagerström Test of Nicotine Dependence; SF-36, Short \\
Form Health Survey-36.
\end{tabular}

\section{Propensity score matching approach}

Baseline characteristics of participants in propensity scorematched sample - the main PSM model included 177 biologically validated reducers; one reducer was excluded because data from the 'Relief from smoking questionnaire' was missing. Of the 176 reducers with complete data, 4 were lost to common support restrictions. Those excluded for this reason were psychologically healthy, with mean (SD) SF-36 scores of 70.0 (28.7). Therefore, 172 reducers were matched to 172 smokers with similar propensity scores (figure 1). Before PSM, there were differences in the proportion of participants receiving active treatment between exposure groups (table 1). After matching, the sample, when measured across trials, was balanced on baseline characteristics (table 2), although there was weak evidence that mental health differed between groups $(\mathrm{p}=0.07)$.

Association between smoking reduction and mental health in the propensity score-matched sample-details of the variables entered into propensity scores and for model adequacy checks are shown in the online supplementary material. After matching, reducers showed a small improvement in mental health of 0.3 (15.9), and this was similar for smokers who continued 0.7 (15.8). There was no clear evidence for a difference between groups. After adjustment for baseline mental health, reducers showed an improvement in mental health compared with continuing smokers; 0.9 (95\% CI -2.2 to 3.9 ; $\mathrm{p}=0.55)$. This association was unchanged after adjustment for age and sex $1.1(95 \%$ CI -2.0 to $4.1, \mathrm{p}=0.50)$.

\section{Comparison of statistical approaches}

Table 3 presents a summary of effect estimates for the two statistical approaches. There was no clear evidence of an association between smoking reduction and mental health using either approach. Moreover, there was no consistent evidence of a difference greater than the MCID $^{\mathrm{i}}$ between exposure groups. However, in all cases, the CIs included the MCID, and the direction of effect indicated that the point estimate differed between the analyses.

${ }^{\mathrm{i}} \mathrm{MCID}=$ difference of 2.0 to 6.2 points between exposure groups 
Table 3 Comparison of statistical approaches

\begin{tabular}{llc}
\hline Approach & $\begin{array}{l}\text { Unadjusted } \\
\text { difference }(95 \% \mathbf{~ C l})\end{array}$ & $\begin{array}{l}\text { Adjusted difference } \\
(95 \% \mathbf{C l})\end{array}$ \\
\hline Regression & $-0.1(-2.5$ to 2.3$)$ & $-0.6(-4.4 \text { to } 3.2)^{*}$ \\
PSM & $0.9(-2.2$ to 3.9$)$ & $1.1(-2.0$ to 4.1$) \dagger$ \\
\hline Reducers were coded as '1' and smokers as '0'. \\
'FTND, treatment status, age, sex and trial. \\
†Age and sex. \\
FTND, Fagerström Test of Nicotine Dependence; PSM, propensity \\
score matching.
\end{tabular}

\section{Risk of bias}

Risk of bias was assessed using an adapted version of the Newcastle-Ottawa Scale for assessment of observational studies. ${ }^{1138}$ The scale assesses risk of bias through ascertainment of exposure and outcome and through loss to follow-up. This study lost one point for high attrition, scoring $4 / 5$ which indicates a low risk of bias (see online supplementary material).

\section{DISCUSSION}

Comparison of regression and PSM approaches indicated there was no statistical evidence of an association between smoking reduction maintained over 12 weeks and change in mental health. The point estimates derived using regression and PSM were close to the null, and the CIs were reasonably precise, albeit just encompassing a clinically important difference in the direction of either an improvement or worsening in mental health.

\section{Strengths and weaknesses}

There were some important strengths to this study. Data were collected following a strict protocol, with biological validation of smoking reduction status. Mental health was assessed using a psychometrically sound tool designed to measure change in general mental health, rather than the presence or absence of mental health disorder. Moreover, participants and raters in the trials were not aware of the study hypothesis, so there were no demand characteristics which may have biased the results. PSM methodology matched participants on factors previously found to predict ability to reduce daily tobacco consumption, and after PSM, a good balance of covariates was achieved and extensive sensitivity analysis showed no evidence that the results were sensitive to the methodological decisions we made. The NewcastleOttawa score suggests that the results were also unlikely to result from multiple sources of bias. A weakness of this study was loss to follow-up, although this rate was similar to other studies of NRT. ${ }^{28}$ It is possible that this introduced bias, ${ }^{39}$ however we suggest this is unlikely. To have biased the study, participants who were lost in the reducer group would have had to have shown the opposite change in mental health to participants who were lost from the continuing smokers group, which seems improbable.

\section{Interpretation}

Results from previous studies have similarly reported a null association; ${ }^{12}{ }^{13}$ however, these studies were imprecise because they used a small sample or a non-validated outcome measure which could also have underestimated the strength of association. This study overcame these issues by use of a larger sample and a validated measure of mental health. As with many observational analyses, our initial approach using regression was at risk of bias through confounding and group membership. The propensity score approach can overcome bias through group membership and offers more control for confounding, ${ }^{20}$ but gave a similar result to the regression modelling in this case. However, the estimates from PSM were a little more imprecise by reducing the effective sample size.

\section{Implications}

Many smokers and clinicians are reluctant to address patients' smoking behaviour for fear that they may cause harm to mental health. ${ }^{40}$ This study adds to the evidence from previous studies that reducing daily cigarette consumption was not associated with change in mental health. $^{12} 13$ Therefore, on average, clinicians can be confident that smoking reduction is unlikely to negatively influence patients' mental health.

\section{CONCLUSION}

If smoking reduction was found to be associated with worsened mental health, this could be due to increasing the duration of withdrawal symptoms, ${ }^{42-45}$ thus supporting the hypothesis that repeated exposure to tobacco can worsen mental health through the withdrawal cycle. $^{43-45}$ Equally, this could indicate that by reducing the frequency of any psychological benefit of tobacco the smoker experiences worse mental health. ${ }^{16}$ However, based on these data analysed in two ways, the evidence here was consistent with previous studies and show no association between smoking reduction and change in mental health, thus suggesting that on average smoking reduction is not any better or worse for mental health than continuing to smoke. Clinicians offering smoking reduction as a route to quit can be confident that on average smoking reduction is not associated with negative change in mental health.

\section{Author affiliations}

${ }^{1}$ Primary Care Clinical Sciences, School of Health \& Population Sciences, University of Birmingham, Birmingham, UK

${ }^{2}$ UK Centre for Tobacco and Alcohol Studies, UK

${ }^{3}$ MRC Integrative Epidemiology Unit (IEU), University of Bristol, Bristol, UK

${ }^{4}$ School of Experimental Psychology, University of Bristol, Bristol, UK

${ }^{5}$ Institute of Psychiatry, King's College London, London, UK

${ }^{6}$ Nuffield Department of Primary Care Health Sciences, The University of Oxford, Oxford, UK

Twitter Follow Gemma Taylor at @GemmaMJTaylor 
Contributors GT contributed towards the study design, analysis and writing the manuscript. AT, MRM, AM and PA contributed to study design, analysis and writing the manuscript.

Funding GT was funded by a National Coordinating Centre for Research Capacity Development scholarship during the conduct of the study. AT is supported by the Medical Research Council (grant number MC_UU_12013/6). MRM has received consulting fees from Servier and grants from Pfizer, outside the submitted work. AM receives funding from the UK Centre for Tobacco and Alcohol Studies. PA reports personal fees from Pfizer, grants and personal fees from McNeil, outside the submitted work. All authors are part of the UK Centre for Tobacco and Alcohol Studies, a UKCRC Public Health Research: Centre of Excellence. Funding from British Heart Foundation, Cancer Research UK, Economic and Social Research Council, Medical Research Council, and the National Institute for Health Research, under the auspices of the UK Clinical Research Collaboration, is gratefully acknowledged.

Competing interests MRM reports grants from Pfizer and non-financial support from GlaxoSmithKline, outside the submitted work. PA reports personal fees from Pfizer, grants and personal fees from AM, outside the submitted work

Provenance and peer review Not commissioned; externally peer reviewed.

Data sharing statement No additional data are available.

Open Access This is an Open Access article distributed in accordance with the Creative Commons Attribution Non Commercial (CC BY-NC 4.0) license, which permits others to distribute, remix, adapt, build upon this work noncommercially, and license their derivative works on different terms, provided the original work is properly cited and the use is non-commercial. See: http:// creativecommons.org/licenses/by-nc/4.0/

\section{REFERENCES}

1. NICE. Tobacco: harm-reduction approaches to smoking (PH45). London, UK: 2013.

2. Pisinger $\mathrm{C}$, Godtfredsen N. Is there a health benefit of reduced tobacco consumption? A systematic review. Nicotine Tob Res 2007;9:631-46.

3. Lindson N, Aveyard P, Hughes J. Reduction versus abrupt cessation in smokers who want to quit. Cochrane Database Syst Rev 2010;(3): CD008033.

4. Physicians RCo, Psychiatrists RCo. Smoking and mental health. London, UK: 2013.

5. Kerr S, Watson $\mathrm{H}$, Tolson $\mathrm{D}$, et al. Smoking after the age of 65 years: a qualitative exploration of older current and former smokers' views on smoking, stopping smoking, and smoking cessation resources and services. Health Soc Care Community 2006;14:572-82.

6. Lawn S, Pols R, Barber J. Smoking and quitting: a qualitative study with community-living psychiatric clients. Soc Sci Med 2002;54:93-104.

7. Thompson B, Thompson A, Thompson J, et al. Heavy smokers: a qualitative analysis of attitudes and beliefs concerning cessation and continued smoking. Nicotine Tob Res 2003;5:923-33.

8. Fidler J, West R. Self-perceived smoking motives and their correlates in a general population sample. Nicotine Tob Res 2009;11:1182-8.

9. McEwen A, West R, McRobbie H. Motives for smoking and their correlates in clients attending Stop Smoking treatment services. Nicotine Tob Res 2008;10:843-50.

10. Ferguson J, Bauld L, Chesterman J, et al. The English smoking treatment services: one-year outcomes. Addiction 2005;100(Suppl 2):59-69.

11. Taylor G, McNeill A, Girling A, et al. Change in mental health after smoking cessation: systematic review and meta-analysis. BMJ 2014;348:g1151.

12. Bolliger CT, Zellweger JP, Danielsson $\mathrm{T}$, et al. Influence of long-term smoking reduction on health risk markers and quality of life. Nicotine Tob Res 2002;4:433-9.

13. Tonnesen $\mathrm{P}$, Pisinger $\mathrm{C}$, Hvidberg $\mathrm{S}$, et al. Effects of smoking cessation and reduction in asthmatics. Nicotine Tob Res 2005;7:139-48.

14. Jarvik ME, Madsen DC, Olmstead RE, et al. Nicotine blood levels and subjective craving for cigarettes. Pharmacol Biochem Behav 2000;66:553-8.

15. Parrott A, Garnham N. Comparative mood states and cognitive skills of cigarette smokers, deprived smokers and nonsmokers. Hum Psychopharmacol Clin Exp 1998;13:367-76.
16. Khantzian E. The self-medication hypothesis of substance use disorders: a reconsideration and recent applications. Harv Rev Psychiatry 1997;4:231-44.

17. Services UDoHaH. The health consequences of smoking: a report of the surgeon general. Atlanta, GA: Centers for Disease Control and Prevention, 2004

18. Boden J, Fergusson D, Horwood J. Cigarette smoking and depression: tests of causal linkages using a longitudinal birth cohort. Br J Psychiatry 2010;196:440-6.

19. Kendler KS, Neale MC, MacLean CJ, et al. Smoking and major depression: a causal analysis. Arch Gen Psychiatry 1993;50:36-43.

20. Rosenbaum $P$, Rubin D. The central role of the propensity score in observational studies for causal effects. Biometrika 1983;70:41-55.

21. Rosenbaum $P$, Rubin D. Reducing bias in observational studies using subclassification on the propensity score. J Am Stat Assoc 1984;79:516-24.

22. Von Elm E, Altman D, Egger M, et al. The Strengthening the Reporting of Observational Studies in Epidemiology (STROBE) statement: guidelines for reporting observational studies. Prev Med 2007;45:247-51.

23. Thoemmes F, Kim E. A systematic review of propensity score methods in the social sciences. Multivariate Behav Res 2011;46:90-118.

24. Batra A, Klingler K, Landfeldt B, et al. Smoking reduction treatment with 4-mg nicotine gum: a double-blind, randomized, placebo-controlled study. Clin Pharmacol Ther 2005;78:689-96.

25. Bolliger $\mathrm{C}$, Zellweger J, Danielsson $\mathrm{T}$, et al. Smoking reduction with oral nicotine inhalers: double blind, randomised clinical trial of efficacy and safety. BMJ 2000;321:329-33.

26. Rennard S, Glover E, Leischow S, et al. Efficacy of the nicotine inhaler in smoking reduction: a double-blind, randomized trial. Nicotine Tob Res 2006;8:555-64.

27. Wennike $\mathrm{P}$, Danielsson $\mathrm{T}$, Landfeldt $\mathrm{B}$, et al. Smoking reduction promotes smoking cessation: results from a double blind, randomized, placebo-controlled trial of nicotine gum with 2-year follow-up. Addiction 2003;98:1395-402.

28. Stead LF, Perera R, Bullen C, et al. Nicotine replacement therapy for smoking cessation. Cochrane Database Syst Rev 2012;11: CD000146.

29. Fagerström KO, Heatherton TF, Kozlowski LT. Nicotine addiction and its assessment. Ear Nose Throat J 1990;69:763-5.

30. Hays R, Sherbourne C, Mazel R. The RAND 36-item health survey 1.0. Health Econ 1993;2:217-27.

31. Hays R, Prince-Embury S, Chen H. RAND-36 Health Status Inventory. San Antonio, TX: The Psychological Corporation, 1998.

32. Hays R, Morales L. The RAND-36 measure of health-related quality of life. Ann Med 2001;33:350-7.

33. Hughes JR, Carpenter MJ. The feasibility of smoking reduction: an update. Addiction 2005;100:1074-89.

34. Vickers A, Altman DG. Statistics notes: analysing controlled trials with baseline and follow up measurements. BMJ 2001;323:1123-4

35. West R, Shiffman S. Effect of oral nicotine dosing forms on cigarette withdrawal symptoms and craving: a systematic review. Psychopharmacology (Berl) 2001;155:115-22.

36. Godtfredsen NS, Prescott E, Osler M, et al. Predictors of smoking reduction and cessation in a cohort of Danish moderate and heavy smokers. Prev Med 2001;33:46-52.

37. Leuven E, Sianesi B. PSMATCH2: STATA module to perform full Mahalanobis and propensity score matching, common support graphing, and covariate imbalance testing. Boston, MA: Boston College Department of Economics, 2003.

38. Wells G, Shea B, O'Connell D, et al. The Newcastle-Ottawa Scale (NOS) for assessing the quality of nonrandomised studies in meta-analyses. Ottawa: 2010.

39. Lachin JM. Statistical considerations in the intent-to-treat principle. Control Clin Trials 2000;21:167-89.

40. McNally L, Oyefeso A, Annan J, et al. A survey of staff attitudes to smoking-related policy and intervention in psychiatric and general health care settings. J Public Health (Oxf) 2006;28:192-6.

41. Johnson JL, Moffat BM, Malchy LA. In the shadow of a new smoke free policy: a discourse analysis of health care providers' engagement in tobacco control in community mental health. Int $J$ Ment Health Syst 2010;4:23.

42. Hughes J. Effects of abstinence from tobacco: valid symptoms and time course. Nicotine Tob Res 2007;9:315-27.

43. Parrott A. Does cigarette smoking cause stress? Am Psychol 1999;54:817-20.

44. Parrott A. Smoking cessation leads to reduced stress, but why? Subst Use Misuse 1995;30:1509-16.

45. Parrott A. Cigarette-derived nicotine is not a medicine. World J Biol Psychiatry 2003;4:49-55. 